\title{
Queue-Aware Subchannel and Power Allocation for Downlink OFDM-Based Cognitive Radio Networks
}

\author{
Long Bao Le, Patrick Mitran, Catherine Rosenberg
}

\begin{abstract}
We investigate downlink resource allocation for OFDM-based cognitive radio networks. It is assumed that secondary users are allowed to transmit on all subchannels as long as the interference they create for primary users remains below a critical threshold. We consider a practical setting where secondary users have finite queue backlogs and a total power constraint at the base station and we perform resource allocation either over one or multiple time slots. Specifically, secondary users with small queue backlogs are only allocated sufficient rates to support their traffic demands and the remaining radio resources are shared among highly backlogged users. Under this setting, we formulate the joint subchannel and power problem with max-min fairness for highly backlogged users. Then, we propose an iterative procedure to find an optimal resource allocation solution using an integer program solver. For online implementation, we develop several heuristics of increasing complexity and performance. Numerical results show that the proposed heuristics achieve very good performance compared to the optimal solutions and that taking queue backlogs into account does not make the heuristics much slower while making the system more responsive to users' need.
\end{abstract}

\section{INTRODUCTION}

Motivated by the fact that spectrum utilization on many frequency bands is very low [1], there has been growing research interest in designing license-exempt systems in which cognitive radio devices can exploit spectrum opportunities in a non-disruptive manner. Research activities include information theoretic aspects of a cognitive radio channel, medium access control (MAC) design, signal processing for sensing, and resource allocation for cognitive radio networks [3]-[7]. In addition, there have been active research/standardization efforts in developing the IEEE 802.22 standard, the first standard for cognitive radio which specifies physical, MAC and air interface for spectrum sharing in the TV broadcast band [2].

In this paper, we consider a downlink resource allocation (RA) problem for an OFDM-based cognitive wireless network comprising one base station (BS) and secondary users (also called CPEs) that can communicate through the BS in single hop. We model the OFDM system as comprising orthogonal subchannels where each subchannel is composed of consecutive subcarriers. We allow the CPEs to transmit on any subchannel as long as the interference they create to primary receivers is below a maximum tolerable limit. Specifically, it is assumed that as a result of distributed spectrum sensing (performed by the BS and CPEs), a vector $T$ is generated which provides the BS with the maximum allowable transmission power it can use on any given subchannel to avoid harmful interference to primary users [8].

Under this setting, we have previously studied in [8] joint subchannel and power allocations that maximize the minimum rate given to any secondary user assuming infinite queue backlogs. We have formulated the problem and developed heuristics to do the resource allocation online with different tradeoffs on efficiency and speed. In this paper, we are interested in the joint subchannel and power allocation considering queue backlogs in order to avoid over-allocation of radio resources to CPEs with small backlogs. Contrary to existing works on traditional or cognitive OFDM resource allocation where the allocation is performed over a single time slot and infinite queue backlogs are usually assumed [9]-[11], we consider a general resource allocation over multiple time slots considering the finite queue backlog of each user. We assume that time is slotted and divided into frames of $L$ time slots. The resource allocation solution is calculated over $F$ time slots $(F=1$ or $1<F \leq L)$, then repeated $k:=L / F$ times in each frame. Note that allocation over multiple time slots captures a practical implementation aspect, improves the granularity of the resource sharing, and is necessary when the number of subchannels is smaller than the number of users or most subchannels are used by primary users with very strict power constraints.

It is clear that the resource allocation problem under consideration is dynamic in nature due to time-varying channel gains, the power constraints given by table $T$ as well as queue backlogs. Clearly, the evolution of user queue backlogs depends on traffic characteristics, available radio resources and a resource allocation strategy. We assume that the channel gains and the vector $T$ remain unchanged over a frame and that the new arrivals of data packets at the BS are only taken into account at the beginning of a frame. Under these assumptions, a resource allocation solution only needs to be recalculated based on the current queue backlog, $T$ and channel gains at the beginning of each frame. In that case, the time to compute the RA solution should be significantly smaller than the duration of a frame which imposes very stringent time constraints on the RA algorithm.

The contributions of our paper are as follows:

- We formulate a resource allocation problem with finite queue backlogs over multiple time slots for the downlink of an OFDM-based cognitive radio network. This is a non-linear problem with integer variables and thus very difficult to solve in general. We propose an iterative procedure to solve it exactly using a commercial integer program solver. This will allow us to validate our heuristics.

- For online implementation, i.e., to compute the allocation in a time significantly lower than the duration of a frame, we develop a family of heuristics that offer different tradeoffs in terms of speed and efficiency. We show that our heuristics perform well in terms of time and efficiency.

- We find that taking queues into consideration has the potential to significantly increase the rate offered to highly backlogged users by not wasting resources on lightly-loaded users, while not increasing substantially the computation time as compared to a family of heuristics that does not take queues into consideration.

- We quantify the performance improvement by performing resource allocation over multiple time slots and find it to be significant even for small values of $F$. 
The remaining of this paper is organized as follows. In Section II, the system model and the downlink resource allocation problem are formulated. The iterative solution approach is presented in Section III. We propose fast heuristics in Section IV. Numerical results are presented in Section V and conclusions are stated in Section VI.

\section{System Model ANd Problem Formulations}

We consider a downlink resource allocation problem which assigns $M$ subchannels to $N$ secondary users (i.e., CPEs) along with the amount of transmitting power that the BS will use on each subchannel. It is assumed that there are $\bar{z}$ possible transmission modes each of which corresponds to one particular modulation and coding scheme. We assume that transmission mode $z$ has rate $R_{z}$ (i.e., $R_{z}$ packets can be sent in a given time-slot) and requires a received Signal to Noise Ratio (SNR) threshold of $\gamma_{z}$ to achieve some desired block error rate.

It is assumed that the BS can use a maximum transmit power of $\bar{P}_{\max }$ in any time slot. Also, due to the distributed sensing outcomes, there is a maximum power constraint $\bar{P}_{j}$ on the power that the BS can use to transmit on subchannel $j$ (i.e., the set of $\bar{P}_{j}$ 's is the previously mentioned vector $T$ ). Let $g_{i j}$ be the channel gain from the BS to CPE $i$ on subchannel $j$ and $f_{i j}(z)$ be the minimum power required to transmit from the BS to CPE $i$ on subchannel $j$ using transmission mode $z$. These quantities are functions of the corresponding channel gain $g_{i j}$, the SNR threshold $\gamma_{z}$ and the noise power at the receivers. We assume that packets to be transmitted are buffered at the BS in separate queues for different CPEs. Let $q_{i}$ be the current backlog for CPE $i$ at the beginning of the frame. Given the backlog information, the radio resources should be allocated to each CPE in such a way that the corresponding allocated rate is just sufficient to support the current backlog whenever possible.

We are interested in finding the joint subchannel, rate, and power allocation for all $N$ CPEs which maximizes the minimum aggregated rate among highly backlogged CPEs (highly backlogged CPEs are CPEs that cannot transmit all of the data in their queues in the current frame). This resource allocation can be performed over $F$ consecutive time slots (called a subframe in the following) where $F=1$ or $1<$ $F \leq L$ and then repeated $k=L / F$ times over the frame.

Note that the formulation of the RA problem without queue backlogs is a straightforward max-min problem and was given in [8]. To formulate the problem with queue backlogs mathematically is less straightforward. We define the binary variable $s_{i j z t}$ as follows: $s_{i j z t}=1$ if subchannel $j$ is allocated to CPE $i$ in time slot $t$, i.e., the BS will transmit to CPE $i$ on this subchannel, using transmission mode $z(1 \leq z \leq \bar{z})$, otherwise $s_{i j z t}=0$. A subchannel can only be allocated to one CPE $i$. The rate offered over a subframe of $F$ timeslots to CPE $i$ can be written as $x_{i}(F):=\sum_{j=1}^{M} \sum_{z=1}^{\bar{z}} \sum_{t=1}^{F} R_{z} s_{i j z t}$ and the total rate over the whole frame is $x_{i}=k \times x_{i}(F)$. We say that a CPE $i$ has its queue fully satisfied if $x_{i} \geq q_{i}$.

Let $S=\left\{s_{i j z t}\right\}$ be a feasible resource allocation over a subframe (and $\mathcal{S}$ be the set of all such feasible RA), i.e., one that satisfies the constraints

$$
\begin{aligned}
& \sum_{i=1}^{N} \sum_{z=1}^{\bar{z}} s_{i j z t} \leq 1, \quad \forall j, t \\
& f_{i j}(z) s_{i j z t} \leq \bar{P}_{j}, \quad \forall i, j, z, t
\end{aligned}
$$

$$
\sum_{i=1}^{N} \sum_{j=1}^{M} \sum_{z=1}^{\bar{z}} f_{i j}(z) s_{i j z t} \leq \bar{P}_{\max }, \quad \forall t
$$

Eq. (1) implies that a given subchannel cannot be allocated to more than one pair $(i, z)$. Eq. (2) refers to the power constraints as given by $T$ while Eq. (3) is the constraint on the total transmit power.

Then let $\Omega(S)$ be the set of CPEs whose queue backlogs are fully satisfied when performing the feasible resource allocation $S$ and $\bar{\Omega}(S)$ be the complement of $\Omega(S)$. Then for each feasible RA, $S$, we can compute the minimum rate received by a CPE in $\bar{\Omega}(S)$ (i.e., whose queue is not entirely satisfied). Our objective is to maximize this minimum over all feasible $S$, i.e.,:

$$
\lambda_{\mathrm{opt}}:=\max _{S \in \mathcal{S}} \min _{i \in \bar{\Omega}(S)} x_{i}(F)
$$

To remove the dependence of the min operation over the set of non-bottleneck CPEs $\bar{\Omega}(S)$, we can write the objective function in an equivalent form as follows:

$$
\max _{S \in \mathcal{S}} \min _{i}\left\{x_{i}(F)+\mu\left(x_{i}(F), q_{i} / k\right)\right\}
$$

where $\mu(x, q)$ is a function which is defined as

$$
\mu(x, q):= \begin{cases}0, & \text { if } x<q \\ \Lambda, & \text { if } x \geq q\end{cases}
$$

where $\Lambda$ is a sufficiently large number. This transformation can be interpreted as follows. For a CPE $i$ such that $x_{i}(F) \geq q_{i} / k$ the objective function for this CPE is large enough that this CPE will not be a bottleneck for the min operation. Therefore, the min in the objective function is only applied to CPEs with queue backlogs that are not met. In the following sections, we will discuss the optimal solution obtained by an integer program solver and also propose suboptimal but fast heuristics for the aforementioned resource allocation problem.

Remark 2.1: The problem formulated above is a very large non-linear problem with integer variables. It is very general and captures several important resource allocation problems. For a traditional OFDM resource allocation problem, constraints (2) should be removed. Also, we can model the corresponding problem with infinite backlog by selecting $\Omega(S)=\emptyset$ for all $S$.

\section{Solution Using An Integer Program Solver}

The objective function of the optimal allocation problem formulated in Eqs. (1)-(3) and (5) is not linear in its optimization variables. Hence its solution cannot be readily obtained by an Integer Program (IP) solver. We now propose an iterative procedure to obtain its solution using a commercial IP solver.

This procedure works by solving a modified problem where each user is required to have either a rate $\lambda_{\text {new }}$ or its queue satisfied. $\lambda_{\text {new }}$ is then iteratively increased until it reaches a maximum $\lambda^{*}$.

Procedure 3.1: Iterative Procedure for Resource Allocation with Finite Queue Backlog

1) Initialization: Set target max-min rates as $\lambda_{\text {old }}=a>0, \lambda_{\text {new }}=$ 0

2) WHILE $\lambda_{\text {new }} \neq \lambda_{\text {old }}$

- Find optimal solution for the following problem

$\max _{s_{i j z t}} \min _{i}\left\{x_{i}(F)+\mu\left(\lambda_{\text {new }}, q_{i} / k\right)\right\}$ 


$$
\begin{aligned}
& \sum_{i=1}^{N} \sum_{z=1}^{\bar{z}} s_{i j z t} \leq 1, \quad \forall j, t \\
& f_{i j}(z) s_{i j z t} \leq \bar{P}_{j}, \quad \forall i, j, z, t \\
& \sum_{i=1}^{N} \sum_{j=1}^{M} \sum_{z=1}^{\bar{z}} f_{i j}(z) s_{i j z t} \leq \bar{P}_{\max }, \quad \forall t \\
& \sum_{j=1}^{M} \sum_{z=1}^{\bar{z}} \sum_{t=1}^{F} R_{z} s_{i j z t} \geq q_{i} / k, \quad \forall i \text { s.t. } q_{i} / k \leq \lambda_{\text {new }}
\end{aligned}
$$

- Update: $\lambda_{\text {old }}=\lambda_{\text {new }}$

- Update: $\lambda_{\text {new }}=\lambda^{*}$ where $\lambda^{*}$ is the optimal objective function of the above optimization problem

END WHILE

We now show that this iterative procedure will find the optimal solution for our resource allocation problem.

Proposition 3.1: The proposed iterative procedure 3.1 converges to the optimal solution of the resource allocation problem formulated in Eqs. (1)-(3) and (5).

Proof: We give only a sketch of the proof due to space limitation. It can be shown that the optimal objective values for the optimization problem (7)-(11) are non-decreasing over consecutive iterations. Because the number of subchannels and the maximum rate on each subchannel are both finite, procedure 3.1 therefore converges.

Now, we show that the converged solution is an optimal solution for the problem formulated in Eqs. (1)-(3) and (5). Let $\lambda_{\text {opt }}$ be the optimal objective value of Eqs. (1)-(3) and (5). Also, suppose that procedure 3.1 converges to $\lambda_{\text {opt }}^{\prime}<\lambda_{\text {opt }}$. This means that substituting either $\lambda_{\text {new }}=\lambda_{\text {opt }}^{\prime}$ or $\lambda_{\text {new }}=\lambda_{\text {opt }}$ into (7)-(11) yields a feasible solution. However, because $\lambda_{\text {opt }}^{\prime}<\lambda_{\text {opt }}$ and $\lambda_{\text {opt }}$ is the optimal solution, any CPE $i$ with $q_{i} / k \leq \lambda_{\text {opt }}^{\prime}$ will receive a rate at least equal to its queue backlog, other CPEs (i.e., those with $q_{i} / k>\lambda_{\text {opt }}^{\prime}$ ) can be supported at rates strictly larger than $\lambda_{\mathrm{opt}}^{\prime}$. This is a contradiction because the solution in the last iteration of procedure 3.1 provides rates of at most $\lambda_{\text {opt }}^{\prime}$ for a CPE $i$ with $q_{i} / k>\lambda_{\text {opt }}^{\prime}$. Hence, our proposed iterative procedure gives the optimal solution.

Even though commercial software like cplex can be used to implement procedure 3.1 corresponding to a system of reasonable size, it usually takes a time that is of the order of tens of seconds to several hours. Practical implementation requires solutions to be calculated in a much shorter time. This motivates us to develop a family of fast heuristics for the aforementioned scenarios in the following section.

\section{FAst Heuristics for Resource Allocation}

Due to the space constraint, we only present here the family of heuristics for the resource allocation problem formulated in (1)-(3) and (5) for $F=1$ and discuss extensions at the end of the section. Therefore we take $k=L$ for now. The first proposed heuristic has two steps. In the first step we allocate power over all subchannels and in step 2 we allocate subchannels sequentially to a CPE whose current allocated rate is the lowest. In the second heuristic, we add a third step which, given the subchannel allocation, performs rate and power allocation, i.e., bit loading. In the last heuristic, we add a fourth step in which we perform some limited perturbations on the solution given by step 3 . We name the heuristics by the last step performed, i.e., step $i$ refers to the heuristic that performs steps 1 to $i$. Finite queue backlogs are taken into account in steps 2, 3 and 4. This avoids over-allocation of radio resources. We provide high level descriptions for all the steps in this section and leave all details in the Appendix except for step 1 which is straightforward.

In step 1, we perform power allocation over the subchannels by sharing $\bar{P}_{\max }$ as uniformly as possible considering the power constraints in vector $T$ (i.e., there is no point allocating more than $\bar{P}_{j}$ to subchannel $j$ ). In fact, it has been shown in [11] that for a traditional OFDM resource allocation problem under a continuous rate assumption that uniform power allocation results in very good performance for a throughput maximization problem.

With the power allocation of step 1 now fixed, we perform subchannel allocation in step 2 (algorithm 2). Specifically, subchannels are allocated to the CPEs sequentially where in each allocation iteration, a CPE with the smallest rate is allocated one subchannel achieving the highest rate given the power allocation in step 1 . Ties in the subchannels are broken in favor of a subchannel with the highest channel gain. Also, ties in minimum rate CPEs are broken in favor of the CPE with

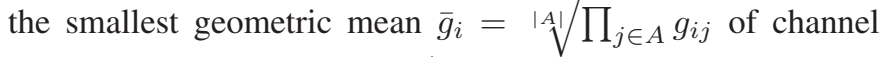
gains calculated over the set $A$ of remaining subchannels (i.e., those not yet allocated). This tie-breaking rule is motivated by the fact that $\bar{g}$ is an indication of the average channel quality for a CPE over the remaining channels.

When a CPE has received enough resources to satisfy its queue, i.e., $q / L$ (since $F=1$ ), it is removed from the list of CPEs, and thus not allocated any more resources. If the "best" subchannel in any allocation iteration does not improve the rate of the CPE to which it is allocated, we allocate all remaining subchannels to CPEs whose queues are not yet fully satisfied in a round robin fashion. This is because the remaining subchannels cannot improve the rate of the "worst" $\mathrm{CPE}$ and we are interested in a max-min. Finally, the power allocated to a subchannel in step 1 is usually larger than the power required to deliver the assigned rate once it has been allocated to a CPE. Therefore, after each subchannel allocation iteration, the residual power on the selected subchannel is calculated and allocated to the set of remaining subchannels as evenly as possible considering the power limits due to $T$.

Given the subchannel allocation solution due to step 2, there is a potential max-min rate improvement by redoing rate and power allocation. This is done by step 3 (Algorithm 3). Let $z_{i j}$ denote the transmission mode on subchannel $j$ for CPE $i$ and define $\Delta P_{i, j}=\left[f_{i j}\left(z_{i j}+1\right)-f_{i j}\left(z_{i j}\right)\right] /\left[R_{z_{i j}+1}-R_{z_{i j}}\right]$ which denotes the extra transmit power per unit rate required to use the next transmission mode for CPE $i$ on subchannel $j$, i.e., to use mode $z_{i j}+1$ instead of mode $z_{i j}$. Specifically, Algorithm 3 sequentially increments the transmission mode of the most power-efficient subchannel for the current minimum rate CPE in each rate update operation. This is a general version of the multi-user bit-loading algorithm. In addition, as soon as a rate of a CPE becomes greater than or equal to its queue backlog, the CPE is removed from the list of active CPEs for all subsequent rate updates.

When Algorithm 3 terminates, the total consumed power may be still well below the maximum power of the BS (i.e., $\left.P<\bar{P}_{\max }\right)$. To exploit the remaining $\mathrm{BS}$ power, in step 4 (Algorithm 4) we perform limited perturbation on the subchannel allocation to improve the minimum rate among all CPEs whose queue is not satisfied. Specifically, for each bottleneck CPE (i.e., a CPE which among all those whose queue is not satisfied, has a minimum rate), we attempt to take one subchannel from a non-bottleneck CPE and allocate 
it to the bottleneck CPE. This subchannel reassignment is only performed if it can improve the rate of the bottleneck CPE while not reducing the subchannel granting CPEs rate to or below the former rate of the bottleneck CPE before perturbation was performed. To verify if a subchannel perturbation satisfies this requirement, we employ a single-user rate and power allocation algorithm to calculate the rates for both bottleneck and granting CPEs. This single-user rate and power allocation is similar in spirit to Algorithm 3 in that we sequentially increment the transmission rate of the most power-efficient subchannel while respecting both individual subchannel power constraints and total BS power limit. This algorithm is, however, for a single user instead of multiple users as in Algorithm 3.

Finally, if the perturbation at the end of Algorithm 4 is successful in increasing the rate of all the bottleneck CPEs from step 3, we proceed to a new round of rate and power allocation (i.e., step 3) followed by perturbation (i.e., step 4). This may be repeated a fixed number of times, or until perturbation fails to increase the rate of a bottleneck CPE.

Remark 4.1: The resource allocation solutions obtained at the end of steps 2, 3, 4 are all feasible. However, the more steps we take the better the performance, in terms of the minimum rate offered to the CPEs whose queue is not satisfied, at the cost of higher computation complexity.

Remark 4.2: The computation penalty for taking queues into account compared to the heuristics specialized to the case of infinite queues is small since it only affects the order of operations in steps 2 and 3. This is confirmed numerically in the results section.

Extensions: We can extend the proposed heuristics to the case $F>1$ by performing power allocation for each of $F$ time slots in step 1 , taking the queue backlog to be $q / k$, and increasing the search space over both subchannels and time slots in steps 2, 3 and 4. For a traditional OFDM resource allocation problem, we can set $\bar{P}_{j}=\infty$ in our proposed heuristics. Finally, for the case of infinite backlog the set of active CPEs always consists of all CPEs. Therefore, the proposed heuristics can be extended to this scenario accordingly.

\section{NUMERICAL RESUltS}

In this section, we investigate the performance of the proposed heuristics and give some insights into RA algorithm design. The inputs for our resource allocation problem are generated as follows. Channel gains from the BS to CPE $i$ for subchannel $j$ are modeled as a combination of path loss and Ricean fading which can be written as $g_{i j}=\left|h_{i j}\right|^{2}\left(d_{0} / d_{i}\right)^{\eta}$, where $h_{i j}$ is an independent Ricean fading gain characterized by its $K$-factor, $\eta$ is the path loss exponent and $d_{0}$ the farfield crossover distance. For simplicity, we calculate $f_{i j}(z)$ as $f_{i j}(z)=\gamma_{z} N_{0} / g_{i j}$ although we could include interference from primary users if needed.

We generate the locations of the $N$ CPEs randomly in a disk of radius $r_{1}$ and generate locations of $N_{p}$ primary receivers randomly in a disk of radius $r_{2}$ centered on the BS. Each primary receiver is randomly assigned one subchannel. For each assigned subchannel $j$, the power limit $\bar{P}_{j}$ is calculated such that the interference power received at the corresponding primary receiver is equal to $\omega N_{0}$ where $N_{0}$ is the noise power. We choose the following parameters in the following. The $K$-factor is $-10 \mathrm{~dB}$ which reflects scenarios with little to no line of sight, $\eta=3, d_{0}=50 \mathrm{~m}, r_{1}=33 \mathrm{~km}, r_{2}=$ $60 \mathrm{~km}$, and $N_{0}=-100 \mathrm{~dB}$. We assume that there are five transmission modes of rates $R_{z}=1,2,3,4,5$ with respective SNR thresholds of $\gamma_{z}=10,14.77,18.45,21.76,24.91 \mathrm{~dB}$. The interference threshold parameter is $\omega=0 \mathrm{~dB}$. To obtain the average heuristic performance and computation time for a given scenario characterized by $\left(M, N, N_{p}\right)$ (recall $M$ is the number of subchannels), we average the corresponding results over 30 independent generations of node positions and fading coefficients.

All our scenarios are with 40 CPEs. The queue sizes are set for the first four groups of CPEs each with 5 CPEs by $q_{i} / L=3,6,9,12$ packets respectively. The queue sizes for the remaining $20 \mathrm{CPEs}$ are set to be $q_{i} / L=30$. We present the performance of the proposed heuristics by showing the average max-min rate for the CPEs whose queue backlogs are not fully satisfied normalized by $L$, i.e., $\lambda / L$, versus $\bar{P}_{\text {max }}$. Because step 3 requires a relatively small increase in computation time but improves the max-min rate quite significantly as compared to step 2, we do not show the performance of step 2 to improve the clarity of figures.

Fig. 1 shows the normalized average max-min rate for the heuristics and the optimal solution computed as discussed in Section III for a scenario with $\left(M, N, N_{p}\right)=(120,40$, 0 ) and subframe sizes $F=1,3$. This scenario corresponds to a traditional OFDM resource allocation problem (similar to IEEE 802.16). Fig. 2 presents results for a scenario with $\left(M, N, N_{p}\right)=(120,40,30)$ and $F=1,3$.

Fig. 1 shows that the difference in the optimal solution between $F=3$ and $F=1$ is rather small for all $\bar{P}_{\max }$ (less than $10 \%)$ while in Fig. 2 it is rather large $(\sim 15 \%)$ for large $\bar{P}_{\text {max }}$. All our heuristics do very well in the case of Fig. 1 (i.e., the difference between the optimal solution and the heuristic's is below 10\%) while in Fig. 2, the difference between the optimal solution and the solution obtained by step 4 (the most efficient heuristic) is of the order of $15 \%$.

In Fig. 3, we show the relative computation time for the setting $\left(M, N, N_{p}\right)=(120,40,30)$ and $F=1,3$ where the computation time of each heuristic is normalized to that of step 2 for $F=1$ at $P_{\max }=50 \mathrm{~W}$. This figure shows that step 3 has relatively small computation time. Step 4 requires moderate computation times at low and moderate values of $P_{\max }$ but this grows quite large for high $P_{\max }$ and $F>1$. We have observed that computation time of step 4 can be significantly reduced by limiting the number of perturbations to a small number. Numerical experiments suggest that running step 4 only two times almost achieves the best performance with reasonable computation times.

Finally, we compare the normalized max-min rates for the CPEs whose queue backlogs are not fully satisfied and the computation times for the case where we take the queue backlogs into account with the case that we call "queueoblivious", where we do not take queues into account and hence we assume that all CPEs are greedy (see [8]). Remember that most of the existing solutions for downlink RA for pure OFDM systems do not take queues into account. The results are given in Table I for the case where $P_{\max }=50 \mathrm{~W}$ (a moderate value of power). This table shows that a significant rate improvement can be achieved by queue-aware heuristics by allocating the resources based on needs. It also shows that the queue-aware heuristics have similar order of computation complexity as the corresponding queue-oblivious ones.

\section{CONCLUSIONS}

We have considered a queue-aware resource allocation problem for OFDM-based cognitive radio networks which is 


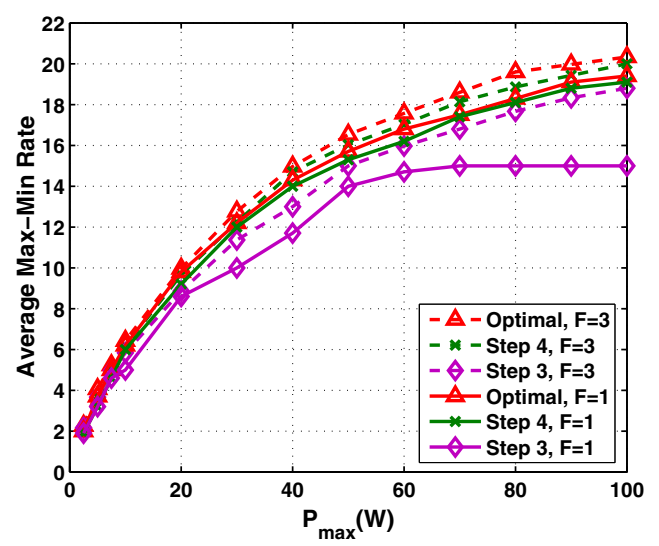

Fig. 1. Normalized average max-min rates obtained by solving the optimization problem and by running the heuristics for $F=1,3,\left(M, N, N_{p}\right)=$ $(120,40,0)$

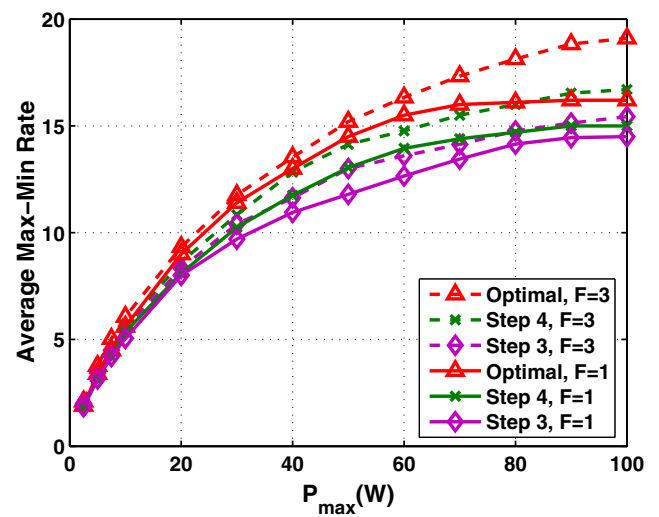

Fig. 2. Normalized average max-min rates obtained by solving the optimization problem and by running the heuristics for $F=1,3,\left(M, N, N_{p}\right)=$ $(120,40,30)$

a non-linear problem with integer variables. We proposed a technique that iteratively computes the optimal solution with the help of a commercial integer program solver. We also designed a family of fast heuristics for online implementation. We investigated the performance of the heuristics through extensive numerical analysis. Our proposed heuristics achieve excellent performance (within $85-90 \%$ of the optimal solution) in all cases. Performing resource allocation over multiple time slots and considering finite queue backlogs were found to significantly enhance network performance. We also found that taking queues into consideration does not increase the computation time as compared to a family of heuristics that does not take queues into consideration.

\section{ApPendiX: Detalled Algorithm Description}

In the following algorithms, $p_{j}$ denotes the allocated power to subchannel $j$ due to step 1 and $h_{i}\left(p_{j}\right)$ denotes the function which gives the maximum achievable rate on subchannel $j$ for CPE $i$ with transmit power $p_{j}$. Note that we take $F=1$ and therefore $k=L$.

\section{Algorithm 2 (Step 2): Subchannel Allocation}

$U \quad$ set of active CPEs

$A \quad$ set of subchannels not yet allocated

$\lambda_{i} \quad$ current rate allocated to CPE $i$

$C_{i} \quad$ set of subchannels allocated to CPE $i$

$\delta p_{j} \quad$ residual power of channel $j$ after its allocation

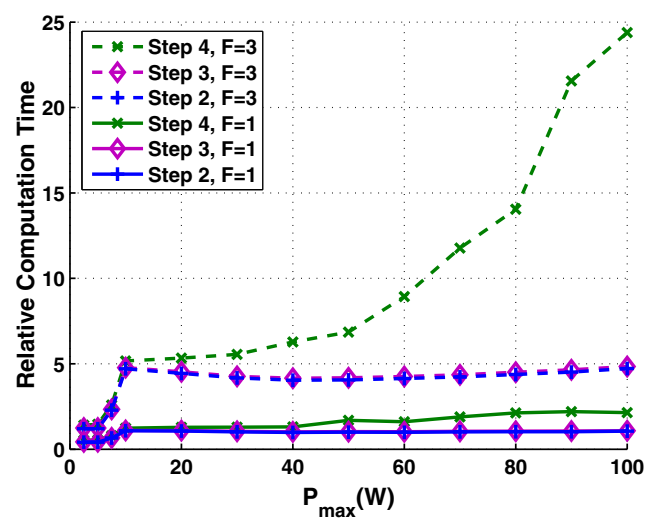

Fig. 3. Relative computation times of the heuristics for $F=1,3$, $\left(M, N, N_{p}\right)=(120,40,30)$ normalized to the computation time of Step 2 for $F=1$ at $P_{\text {max }}=50 \mathrm{~W}$

TABLE I

QueUe-AWARE VS. QUEUE-OBLIVIOUS HEURISTICS FOR $P_{\max }=50 \mathrm{~W}$

\begin{tabular}{|c|c|c|c|}
\hline \multicolumn{4}{|c|}{$\left(M, N, N_{p}\right)=(120,40,0), F=1$} \\
\hline \multicolumn{2}{|c|}{ Queue-Aware } & \multicolumn{2}{|c|}{ Queue-Oblivious } \\
\hline Step 3-Rate & Step 4-Rate & Step 3-Rate & Step 4-Rate \\
\hline 14.40 & 14.93 & 10.00 & 10.73 \\
\hline Step 3-Time & Step 4-Time & Step 3-Time & Step 4-Time \\
\hline 0.221 & 0.463 & 0.236 & 0.328 \\
\hline \multicolumn{4}{|c|}{$\left(M, N, N_{p}\right)=(120,40,0), F=3$} \\
\hline \multicolumn{2}{|c|}{ Queue-Aware } & \multicolumn{2}{|c|}{ Queue-Oblivious } \\
\hline Step 3-Rate & Step 4-Rate & Step 3-Rate & Step 4-Rate \\
\hline 14.60 & 16.13 & 11.46 & 11.63 \\
\hline Step 3-Time & Step 4-Time & Step 3-Time & Step 4-Time \\
\hline 0.930 & 1.370 & 1.032 & 1.301 \\
\hline \multicolumn{4}{|c|}{$\overline{\left(M, N, N_{p}\right)=(120,40,30), F=1}$} \\
\hline \multicolumn{2}{|c|}{ Queue-Aware } & \multicolumn{2}{|c|}{ Queue-Oblivious } \\
\hline Step 3-Rate & Step 4-Rate & Step 3-Rate & Step 4-Rate \\
\hline 11.50 & 12.53 & 9.77 & 9.93 \\
\hline Step 3-Time & Step 4-Time & Step 3-Time & Step 4-Time \\
\hline 0.210 & 0.323 & 0.218 & 0.296 \\
\hline \multicolumn{4}{|c|}{$\left(M, N, N_{p}\right)=(120,40,30), F=3$} \\
\hline \multicolumn{2}{|c|}{ Queue-Aware } & \multicolumn{2}{|c|}{ Queue-Oblivious } \\
\hline Step 3-Rate & Step 4-Rate & Step 3-Rate & Step 4-Rate \\
\hline 13.12 & 14.34 & 10.29 & 10.44 \\
\hline Step 3-Time & Step 4-Time & Step 3-Time & Step 4-Time \\
\hline 0.898 & 1.455 & 0.984 & 1.172 \\
\hline
\end{tabular}

1) Initialization: saturation $=0$ and $\lambda_{i}=0$ for $i \in\{1,2, \ldots, N\}$, $A=\{1,2, \ldots, M\}, U=\{1,2, \ldots, N\}$, $C_{i}=\emptyset$ for $i \in\{1,2, \ldots, N\}$

2) WHILE $|U|>0$

a) IF saturation $=0$

- Find $i^{*}$ such that $i^{*}=\operatorname{argmin}_{i \in U} \lambda_{i}$ ELSE

- Choose $i^{*}$ as the next CPE in set $U$ END IF

b) IF $\max _{\{j \in A\}}\left\{h_{i *}\left(p_{j}\right)\right\}>0$

- Find the set of subchannels with maximum rate: $\Psi=\left\{j \mid h_{i^{*}}\left(p_{j}\right)=\max _{\{j \in A\}}\left\{h_{i^{*}}\left(p_{j}\right)\right\}\right\}$

- Allocate subchannel $j^{*}$ for CPE $i^{*}: C_{i^{*}}=C_{i^{*}}+\left\{j^{*}\right\}$, where $j^{*}=\operatorname{argmax}_{\{j \in \Psi\}}\left\{g_{i^{*} j}\right\}$

- Update $A=A-\left\{j^{*}\right\}, \lambda_{i^{*}}=\lambda_{i^{*}}+h_{i^{*}}\left(p_{j^{*}}\right)$

- IF $\lambda_{i^{*}} \geq q_{i^{*}} / L$, update: $U=U-\left\{i^{*}\right\}$

ELSE IF $h_{i}\left(p_{j^{*}}\right)=0$

- Update: saturation $=1$

- Find subchannel $j^{*}$ : $j^{*}=\operatorname{argmax}_{\{j \in A\}}\left\{g_{i^{*} j} \bar{P}_{j}\right\}$ 
- $\operatorname{IF} h_{i^{*}}\left(\bar{P}_{j^{*}}\right)>0$

- Allocate subchannel and update: $C_{i^{*}}=C_{i^{*}}+\left\{j^{*}\right\}$, $A=A-\left\{j^{*}\right\}$

ELSE IF $h_{i^{*}}\left(\bar{P}_{j^{*}}\right)=0$

- Remove CPE $i^{*}$ from active list: $U=U-\left\{i^{*}\right\}$

END IF

END IF

c) Perform residual power distribution

- Calculate residual power on subchannel $j^{*}$ as $\delta p_{j^{*}}=$ $p_{j^{*}}-f_{i^{*} j^{*}}\left(h_{i^{*}}\left(p_{j^{*}}\right)\right)$

- Distribute this residual power over the set $A$

\section{END WHILE}

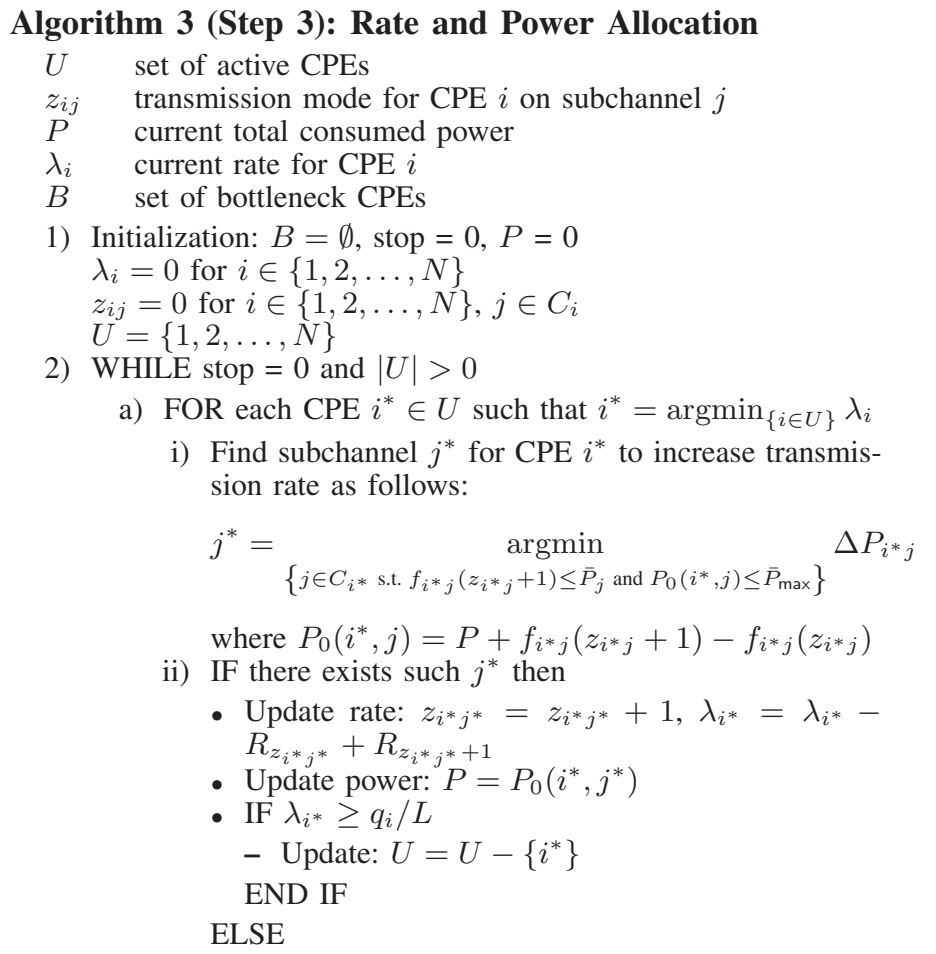

- Update: $B=B+\left\{i^{*}\right\}$, stop $=1$

END IF

END FOR

END WHILE

\section{Algorithm 4 (Step 4): Perturbation}

$\lambda \quad$ min rate after step 3

$B \quad$ set of bottleneck CPEs from step 3

$U$ set of active CPEs

$D \quad$ current set of non-bottleneck CPEs

$P_{1} \quad$ temporary total consumed power

$P_{2} \quad$ another temporary total consumed power

1) Initialization: $\lambda=\min _{i}\left\{\lambda_{i}\right\}$

2) FOR each $b \in B$

a) Update current set of non-bottleneck CPEs:

$D=\{1,2, \cdots, M\}-B$ and initialize stop $=0$

b) WHILE $D \neq \emptyset$ and stop $=0$

i) Find one non-bottleneck CPE: $i^{*}=\operatorname{argmax}_{\{i \in D\}}\left|C_{i}\right|$

ii) Initialization: $C=C_{i}$

iii) WHILE $|C|>0$ and stop $=0$

- Find one subchannel in $C$ for reallocation: $j^{*}=\operatorname{argmin}_{\{j \in C\}}\left\{g_{i^{*} j} \bar{P}_{j}\right\}$

- Temporarily take subchannel $j^{*}$ from CPE $i^{*}$ and assign it to CPE $b$

- Find the new rate for CPE $b$ given initial consumed power $P$ using a single-user rate and power allocation algorithm to obtain new rate $r_{b}$, total consumed power $P_{1}$, and $z_{b j}^{\prime}$ for $j \in C_{b}$

- IF $r_{b}>\lambda$
- Find the new rate for CPE $i^{*}$ given initial consumed power $P_{1}$ using single-user rate and power allocation algorithm to obtain new rate $r_{i^{*}}$, total consumed power $P_{2}$, and $z_{i^{*} j}$ for $j \in C_{i^{*}}$

- IF $r_{i^{*}}>\lambda$ or $r_{i^{*}} \geq q_{i^{*}} / L$ and $P_{2} \leq \bar{P}_{\max }$

* Update subchannel allocation:

$C_{i^{*}}=C_{i^{*}}-\left\{j^{*}\right\}, C_{b}=C_{b}+\left\{j^{*}\right\}$

* Update rates for CPE $b$ and $i^{*}$ :

$\lambda_{b}=r_{b}, \lambda_{i *}=r_{i}$

* Update subchannel rates for CPE $b$ and $i^{*}$ : $z_{b j}=z_{b j}^{\prime}, z_{i^{*} j}=z_{i^{*} j}$ for all $j$

* Update: $P=P_{2}, B=B-\{b\}$

* IF $r_{b} \geq q_{b} / L$ and/or $r_{i^{*}} \geq q_{i^{*}} / L$, remove corresponding CPE from the list of active CPEs: $U=U-\{b\}$ and/or $U=U-\left\{i^{*}\right\}$

* Set stop $=1$

ELSE IF $r_{i^{*}} \leq \lambda$

* Update: $C=C-\left\{j^{*}\right\}$

END IF

ELSE IF $r_{b} \leq \lambda$

- Remove subchannel $j^{*}$ from set $C$ : $C=C-\left\{j^{*}\right\}$

END IF

END WHILE

iv) IF $|C|=0$

- Remove CPE $i^{*}$ from set $D: D=D-\left\{i^{*}\right\}$

END IF

END WHILE

END FOR

3) IF $B \neq \emptyset$

- Terminate the perturbation.

ELSE

- Set stop $=0$ and run the rate and power allocation algorithm in step 3 starting from 2).

END IF

\section{REFERENCES}

1] FCC Spectrum Policy Task Force, "Report of the spectrum efficiency working group," FCC, Tech. Rep., Nov. 2002.

[2] C. Cordeiro, K. Challapali, D. Birru, and N. S. Shankar, "IEEE 802.22: The first worldwide wireless standard based on cognitive radios," in Proc. DySpan 2005, Nov. 2005.

[3] N. Devroye, P. Mitran and V. Tarokh, "Achievable rates in cognitive radio channels," IEEE Trans. Inf. Theory, vol. 52, no. 5, pp. 1813-1827, 2006.

[4] M. Gandetto and C. Regazzoni, "Spectrum sensing: A distributed approach for cognitive terminals," IEEE J. Sel. Areas Commun., vol. 25, no. 3, pp. 546-557, April 2007.

[5] L. Le and E. Hossain, "QoS-aware spectrum sharing in cognitive wireless networks," in Proc. IEEE GLOBECOM'2007, Nov. 2007.

[6] P. Wang, M. Zhao, L. Xiao, S. Zhou, and J. Wang, "Power allocation in OFDM-based cognitive radio systems," in IEEE GLOBECOM, 2007.

[7] H. Wendong, D. Willkomm, M. Abusubaih, J. Gross, G. Vlantis, M. Gerla, and A. Wolisz, "Dynamic frequency hopping communities for efficient IEEE 802.22 operation," IEEE Commun. Mag., pp. 80-87, vol. 45, no. 5, May 2007.

[8] P. Mitran, L. Le, C. Rosenberg and A. Girard, "Resource allocation for downlink spectrum sharing in cognitive radio networks," in Proc. IEEE VTC-Fall 08, Sept. 2008

[9] C. Y. Wong, R. S. Cheng, and K. B. Letaief, and R. D. Murch, "Multiuser OFDM with adaptive subcarrier, bit, and power allocation," IEEE J. Sel. Areas Commun., vol. 17, no. 10, pp. 1743-1758, Oct. 1999.

[10] D. Kivanc, G. Li, and H. Liu, "Computationally efficient bandwidth allocation and power control for OFDMA," IEEE Trans. Wireless Commun. vol. 2 , no. 6, pp. 1150-1158, Nov. 2003.

[11] W. Rhee and J. M. Cioffi, "Increase in capacity of multiuser OFDMA system using dynamic subchannel allocation," in Proc. IEEE VTC'2000. 